\title{
Focus issue on polyphenols
}

\author{
Veronique Cheynier $^{1} \cdot$ Kumi Yoshida ${ }^{2} \cdot$ Stefan Martens $^{3}$
}

Published online: 8 August 2015

(c) Springer-Verlag Berlin Heidelberg 2015

Polypenols are the major group of plant secondary metabolites, structurally extremely diverse and involved in numerous functions in plants. Among them, polymers, such as lignin, which is essential for the structure and for a physical barrier of higher plants, and tannins, involved in plant defense, are particularly important both quantitatively and qualitatively. Other classes include flavonoid subgroups such as anthocyanins (i.e. red and blue pigments, playing a role in plant pollination and defense), flavonols (yellow pigments involved in UV protection) or isoflavones, and non-flavonoids, e.g. lignans, stilbenoids, curcuminoids, etc.

All these molecules are important dietary constituents, due to their occurrence in fruits, vegetables, tea, wine, chocolate, spices, etc., and attract considerable interest because of their potential benefit for human health. Moreover, they find new applications for the development of bio-based materials.

Polyphenol research, expanding to simpler plant phenolics such as phenolic acids, is very active, covering a

Stefan Martens

stefan.martens@fmach.it

Veronique Cheynier

cheynier@supagro.inra.fr

Kumi Yoshida

yoshidak@is.nagoya-u.ac.jp

1 INRA, UMR1083 Sciences pour l'Oenologie, 34060 Montpellier, France

2 Graduate School of Information Science, Nagoya University, Nagoya, Japan

3 Department of Food Quality and Nutrition, Centro Ricerca e Innovazione, Fondazione Edmund Mach, Via E. Mach, 1, 38010 San Michele all'Adige, TN, Italy wide range of topics and areas. Promoting this research is the aim of Groupe Polyphenols, an International Society founded in 1972 (http://www.groupepolyphenols.com/). This is achieved in particular through the organization of the bi-annual International Conferences on Polyphenols (ICP) and edition of Recent Advances in Polyphenol Research (RAPR), containing chapters written by ICP plenary lecturers and other invited contributors selected to convey the most significant advances in the field. Another series of conferences, the Tannin conferences with a specific focus on tannin research, has been initiated in 1988 by Pr. R. Hemingway and is held periodically since.

The XXVIIth International Conference on Polyphenols and the 8th Tannin Conference have been held jointly for the first time in their history in Nagoya, Japan, in September 2014. This joint meeting has provided scientists of both communities worldwide (35 countries represented) with a unique forum to exchange ideas and present novel findings on plant polyphenol research, including biosynthesis, genetics and metabolic engineering, functions in plants and ecosystems, physical-chemical properties and biological properties related to health benefits as well as various applications in food, nutrition, natural medicine, or material science. Some of the cutting-edge contributions are presented in this issue of Planta. 